\title{
Periodic solutions of Lienard differential equations via averaging theory of order two
}

\author{
JAUME LLIBRE ${ }^{1}$, DOUGLAS D. NOVAES ${ }^{2}$ and MARCO A. TEIXEIRA ${ }^{2}$ \\ ${ }^{1}$ Departament de Matematiques, Universitat Autònoma de Barcelona, 08193 Bellaterra, Barcelona, Catalonia, Spain \\ ${ }^{2}$ Departamento de Matemática, Universidade Estadual de Campinas, Rua Sérgio Buarque de Holanda, 651, Cidade Universitária \\ Zeferino Vaz, 13083-859 Campinas, SP, Brasil
}

Manuscript received on March 21, 2014; accepted for publication on February 20, 2015

\begin{abstract}
For $\varepsilon \neq 0$ sufficiently small we provide sufficient conditions for the existence of periodic solutions for the Lienard differential equations of the form

$$
x^{\prime \prime}+f(x) x^{\prime}+n^{2} x+g(x)=\varepsilon^{2} p_{1}(t)+\varepsilon^{3} p_{2}(t),
$$

where $n$ is a positive integer, $f: \mathbb{R} \rightarrow \mathbb{R}$ is a $C^{3}$ function, $g: \mathbb{R} \rightarrow \mathbb{R}$ is a $C^{4}$ function, and $p_{i}: \mathbb{R} \rightarrow \mathbb{R}$ for $i=1,2$ are continuous $2 \pi$-periodic function. The main tool used in this paper is the averaging theory of second order. We also provide one application of the main result obtained.
\end{abstract}

Key words: periodic solution, Lienard differential equation, averaging theory, bifurcation theory.

\section{1 - INTRODUCTION AND STATEMENT OF THE MAIN RESULTS}

In Ma and Wang (2013) it was studied the existence of periodic solutions for the class of Lienard differential equations of the form

$$
x^{\prime \prime}+f(x) x^{\prime}+V^{\prime}(x)+g(x)=p(t),
$$

where $f, V, g, p: \mathbb{R} \rightarrow \mathbb{R}$ are continuous and $p$ is $2 \pi$-periodic. They also assumed that $g$ and $V^{\prime}$ are locally Lipschitz; and the function $V$ is a $2 \pi / n$-isochronous potential, i.e. all nontrivial solutions of $x^{\prime \prime}+V^{\prime}(x)=0$ are $2 \pi / n$-periodic, where $n$ is a positive integer. The authors provided sufficient bounded conditions related with the functions involved in equation (1) to ensure the existence of periodic solutions for this equation. We shall study a particular subclass of equations (1) in which such bounded conditions are not necessary.

In this paper we consider the subclass

$$
x^{\prime \prime}+f(x) x^{\prime}+n^{2} x+g(x)=\varepsilon^{2} p_{1}(t)+\varepsilon^{3} p_{2}(t),
$$

AMS (2010): 37G15, 37C 80, 37C30

Correspondence to: Douglas D. Novaes

E-mail: ddnovaes@ime.unicamp.br 
of Lienard differential equations (1) where $n$ is a positive integer, $\varepsilon$ is a small parameter, $f: \mathbb{R} \rightarrow \mathbb{R}$ is a $\mathcal{C}^{3}$ function in a neighborhood of $x=0, g: \mathbb{R} \rightarrow \mathbb{R}$ is a $\mathcal{C}^{4}$ function in a neighborhood of $x=0$, and $p_{i}: \mathbb{R} \rightarrow \mathbb{R}$ for $i=1,2$ are continuous $2 \pi$-periodic functions. Note that here we are taking $V(x)=n^{2} x^{2} / 2$ which is a $2 \pi / n$-isochronous potential, already considered in Ma and Wang (2013).

The objective of this paper is to give sufficient conditions on the functions $f, g$ and $p_{i}$ to assure the existence of periodic solutions for the equation (2). Here the functions $f$ and $g$ do not need to satisfy the bounded conditions of Ma and Wang (2013).

In general, to obtain analytically periodic solutions of a differential system, is a very difficult problem, many times impossible. Here using the averaging theory this difficult problem for the differential equations (2) is reduced to find the zeros of a nonlinear system of two functions with two unknowns. We must mention that the averaging theory for finding periodic solutions, in general, does not provide all the periodic solutions of the system. More precisely, the main tool used will be the averaging theory of second order for computing periodic orbits, see Llibre (2002), Buică and Llibre (2004), Giné et al. (2013), Llibre et al. (2014). This theory provides a quantitative relation between the solutions of some non-autonomous periodic differential system and the solutions of the averaged differential system, which is autonomous. In this way a finite dimensional function $f$ is computed, the simple zeros of this function correspond with the periodic orbits of the non-autonomous periodic differential system for values of a parameter $\varepsilon \neq 0$ sufficiently small. Here a simple zero $a$ of a function $f$ means that the Jacobian of $f$ at $a$ is not zero. For a general introduction to the averaging theory see, for instance, the book Sanders et al. (2007).

In order to present our results we need some preliminary definitions and notations. We define the constants

$$
\alpha=\int_{0}^{2 \pi} p_{1}(t) \sin (n t) d t \quad \text { and } \quad \beta=\int_{0}^{2 \pi} p_{1}(t) \cos (n t) d t
$$

and the two functions

$$
\begin{aligned}
f_{21}(u, v)= & -\frac{n^{2} u^{2}+v^{2}}{24 n^{6}}\left(3 n^{2}\left(a_{1} b_{1}-a_{2} n^{2}\right) u+\left(10 b_{1}^{2}+n^{2}\left(a_{1}^{2}-9 b_{2}\right)\right) v\right) \\
& -\frac{1}{2 \pi n^{3}} \int_{0}^{2 \pi} \sin (n t)\left(\left(\int_{0}^{t} p_{1}(s) \cos (n s) d s\right)\right. \\
& \cdot\left(-b_{1} v+\left(n^{2} a_{1} u+b_{1} v\right) \cos (2 n t)+n\left(a_{1} v-b_{1} u\right) \sin (2 n t)\right) \\
& +n\left(n p_{2}(t)+\left(\int_{0}^{t} \frac{p_{1}(s) \sin (n s)}{n} d s\right)\right. \\
& \left.\left.\cdot\left(n b_{1} u+n\left(b_{1} u-a_{1} v\right) \cos (2 n t)+\left(n^{2} a_{1} u+b_{1} v\right) \sin (2 n t)\right)\right)\right) d t
\end{aligned}
$$


and

$$
\begin{aligned}
f_{22}(u, v)= & \frac{n^{2} u^{2}+v^{2}}{24 n^{4}}\left(\left(10 b_{1}^{2}+n^{2}\left(a_{1}^{2}-9 b_{2}\right)\right) u+\left(3 n^{2} a_{2}-3 a_{1} b_{1}\right) v\right) \\
& +\frac{1}{2 \pi n^{2}} \int_{0}^{2 \pi}\left(n^{2} p_{2}(t) \cos (n t)+\cos (n t)\left(\int_{0}^{t} \frac{p_{1}(s) \sin (n s)}{n} d s\right)\right. \\
& \cdot n\left(n b_{1} u+n\left(b_{1} u-a_{1} v\right) \cos (2 n t)+\left(n^{2} a_{1} u+b_{1} v\right) \sin (2 n t)\right) \\
& +\cos (n t)\left(\int_{0}^{t} p_{1}(s) \cos (n s) d s\right) \\
& \left.\cdot\left(-b_{1} v+\left(n^{2} a_{1} u+b_{1} v\right) \cos (2 n t)+n\left(a_{1} v-b_{1} u\right) \sin (2 n t)\right)\right) d t,
\end{aligned}
$$

where

$$
a_{1}=f^{\prime}(0), \quad a_{2}=\frac{1}{2} f^{\prime \prime}(0), \quad b_{1}=\frac{1}{2} g^{\prime \prime}(0), \quad \text { and } \quad b_{2}=\frac{1}{6} g^{\prime \prime \prime}(0) .
$$

Our main result is the following.

Theorem 1. Assume that the functions $f$ and $g$ of the Lienard differential equation (2) satisfy that $f$ is a $\mathcal{C}^{3}$ function in a neighborhood of $x=0, g$ is a $\mathcal{C}^{4}$ function in a neighborhood of $x=0$, and $f(0)=g(0)=$ $g^{\prime}(0)=0$. Suppose also that the constants $\alpha=\beta=0$. Then for $\varepsilon \neq 0$ sufficiently small and for every simple zero $\left(u^{*}, v^{*}\right)$ of the system

$$
f_{21}(u, v)=0, \quad f_{22}(u, v)=0
$$

there exists a periodic solution $x(t, \varepsilon)$ of the differential equation (2) such that $x(0, \varepsilon) \approx \varepsilon u^{*}+\mathcal{O}\left(\varepsilon^{2}\right)$ and $x^{\prime}(0, \varepsilon) \approx \varepsilon v^{*}+\mathcal{O}\left(\varepsilon^{2}\right)$.

Theorem 1 is proved in section 2 .

In the next corollary we apply Theorem 1 to a given Lienard differential equation (2) and we show that such an equation has two periodic solutions.

Corollary 2. Assume that

$$
f(x)=a_{1} x+a_{2} x^{2}, \quad g(x)=b_{1} x^{2}+b_{2} x^{3}, \quad p_{1}(t)=1, \text { and } p_{2}(t)=\sin (n t),
$$

where

$$
\begin{aligned}
& a_{1}=\frac{-7 n^{5}+2 n^{3}+n}{-6 n^{4}-4 n^{2}+2}, \\
& a_{2}=\frac{n\left(7 n^{7}-6 n^{6}-2 n^{5}+2 n^{4}-n^{3}+6 n^{2}-2\right)}{\left(n^{2}+1\right)^{2}\left(3 n^{2}-1\right)^{2}}, \\
& b_{1}=\frac{2 n^{5}}{3 n^{4}+2 n^{2}-1}, \text { and } \\
& b_{2}=\frac{n^{2}\left(49 n^{8}+132 n^{6}-144 n^{5}-10 n^{4}-96 n^{3}+4 n^{2}+48 n+1\right)}{36\left(n^{2}+1\right)^{2}\left(3 n^{2}-1\right)^{2}} .
\end{aligned}
$$


Then, for $\varepsilon \neq 0$ sufficiently small the Lienard differential equation (2) has two periodic solutions $x_{i}(t, \varepsilon)$ for $i=1,2$ such that

$$
\begin{aligned}
& x_{1}(0, \varepsilon) \approx \varepsilon+\mathcal{O}\left(\varepsilon^{2}\right) \quad \text { and } \quad x_{1}^{\prime}(0, \varepsilon) \approx \varepsilon+\mathcal{O}\left(\varepsilon^{2}\right), \\
& x_{2}(0, \varepsilon) \approx 2 \varepsilon+\mathcal{O}\left(\varepsilon^{2}\right) \quad \text { and } \quad x_{2}^{\prime}(0, \varepsilon) \approx \mathcal{O}\left(\varepsilon^{2}\right) .
\end{aligned}
$$

Corollary 2 is proved in section 2.

In Appendix we summarize the averaging theory of second order, for studying periodic solutions that we shall need for proving Theorem 1 .

We remark that in Ma and Wang (2013) the results about periodic solutions of system (1) are given, among other hypotheses, under the assumption that the function

$$
F(x)=\int_{0}^{x} f(s) d s
$$

is bounded on $\mathbb{R}$ or that

$$
-\infty<\liminf _{|x| \rightarrow+\infty} \frac{F(x)}{|x|^{p-2} x} \leq \lim _{|x| \rightarrow+\infty} \frac{F(x)}{|x|^{p-2} x}<+\infty,
$$

for some $1 \leq p<2$. It is worth emphasizing that Theorem 1 assures the existence of periodic solutions of system (2) without making any bounding assumption. Consequently, the present result detects some periodic solutions which are not detected by the theorems given in Ma and Wang (2013). Indeed, concerning the Corollary $2, F(x)=a_{1} x^{2} / 2+a_{2} x^{3} / 3$. It is easy to see that the above conditions do not hold in this case.

\section{2 - PROOF OF THE RESULTS}

In this section we shall prove Theorem 1 and Corollary 2.

Proof of Theorem 1. First we shall write the Lienard differential equations (2) in the normal form for applying the averaging theory, see Theorem 3 of the Appendix.

We change the variable $x$ by a new variable $z$ doing the rescaling $x=\varepsilon z$. Then equation (3) becomes

$$
z^{\prime \prime}+f(\varepsilon z) z^{\prime}+n^{2} z+\frac{g(\varepsilon z)}{\varepsilon}=\varepsilon p_{1}(t)+\varepsilon^{2} p_{2}(t) .
$$

Since $f$ is a $\mathcal{C}^{3}$ function in a neighborhood of $x=0, g$ is a $\mathcal{C}^{4}$ function in a neighborhood of $x=0$, and $f(0)=g(0)=g^{\prime}(0)=0$, we can write

$$
\begin{aligned}
& f(\varepsilon z)=\varepsilon f^{\prime}(0) z+\varepsilon^{2} \frac{1}{2} f^{\prime \prime}(0) z^{2}+\mathcal{O}\left(\varepsilon^{3}\right)=\varepsilon a_{1} z+\varepsilon^{2} a_{2} z^{2}+\mathcal{O}\left(\varepsilon^{3}\right), \\
& g(\varepsilon z)=\varepsilon^{2} \frac{1}{2} g^{\prime \prime}(0) z^{2}+\varepsilon^{3} \frac{1}{6} g^{\prime \prime \prime}(0) z^{3}+\mathcal{O}\left(\varepsilon^{4}\right)=\varepsilon^{2} b_{1} z^{2}+\varepsilon^{3} b_{2} z^{3}+\mathcal{O}\left(\varepsilon^{4}\right) .
\end{aligned}
$$

Thus

$$
f(\varepsilon z) z^{\prime}+\frac{g(\varepsilon z)}{\varepsilon}=\varepsilon\left(a_{1} z z^{\prime}+b_{1} z^{2}\right)+\varepsilon^{2}\left(a_{2} z^{2} z^{\prime}+b_{2} z^{3}\right)+\mathcal{O}\left(\varepsilon^{3}\right) .
$$


We introduce a new variable $w=z^{\prime}$. Then the differential equation (3) can be written as the differential system

$$
\begin{aligned}
z^{\prime}= & w \\
w^{\prime}= & -n^{2} z+\varepsilon\left(p_{1}(t)-a_{1} z w-b_{1} z^{2}\right)+\varepsilon^{2}\left(p_{2}(t)-a_{2} z^{2} w-b_{2} z^{3}\right) \\
& +\mathcal{O}\left(\varepsilon^{3}\right) .
\end{aligned}
$$

Now we change the variables $(z, w)$ by the new variables $(u, v)$ defined through the equality

$$
\left(\begin{array}{c}
z \\
w
\end{array}\right)=\left(\begin{array}{cc}
\cos (n t) & \frac{\sin (n t)}{n} \\
-n \sin (n t) & \cos (n t)
\end{array}\right)\left(\begin{array}{l}
u \\
v
\end{array}\right) .
$$

We do these changes so that the differential system in the new variables $(u, v)$ starts with terms of order $\mathcal{O}(\varepsilon)$ and we can apply the averaging theory described in the Appendix. Thus the differential system (4) in the new variables becomes

$$
\begin{aligned}
u^{\prime} & =\varepsilon \frac{1}{n^{3}} G_{1} \sin (n t)+\varepsilon^{2} \frac{1}{n^{4}} G_{2} \sin (n t)+\mathcal{O}\left(\varepsilon^{3}\right) \\
& =\varepsilon F_{11}(t, u, v)+\varepsilon^{2} F_{21}(t, u, v)+\mathcal{O}\left(\varepsilon^{3}\right), \\
v^{\prime} & =\varepsilon \frac{1}{n^{3}} G_{1} \cos (n t)+\varepsilon^{2} \frac{1}{n^{4}} G_{2} \cos (n t)+\mathcal{O}\left(\varepsilon^{3}\right) \\
& =\varepsilon F_{12}(t, u, v)+\varepsilon^{2} F_{22}(t, u, v)+\mathcal{O}\left(\varepsilon^{3}\right),
\end{aligned}
$$

where

$$
\begin{aligned}
G_{1} & =-n^{2} p_{1}(t)+A\left(B_{1} \cos (n t)+C_{1} \sin (n t)\right) \\
G_{2} & =-n^{3} p_{2}(t)+A^{2}\left(B_{2} \cos (n t)+C_{2} \sin (n t)\right) \\
A & =n u \cos (n t)+v \sin (n t), \\
B_{i} & =n\left(b_{i} u+a_{i} v\right) \\
C_{i} & =-n^{2} a_{i} u+b_{i} v
\end{aligned}
$$

In short, the differential system (6) is in the normal form for applying the averaging theory described in Theorem 3 of the Appendix. Using the notation of the Appendix we have $T=2 \pi$. 
Let $F_{i}(t, u, v)=\left(F_{i 1}(t, u, v), F_{i 2}(t, u, v)\right)$ for $i=1,2$. Now we compute the function $f_{1}(u, v)$ defined in the Appendix and we get, from the assumptions, that

$$
\begin{aligned}
f_{1}(u, v) & =\frac{1}{2 \pi}\left(\int_{0}^{2 \pi} F_{11}(t, u, v) d t, \int_{0}^{2 \pi} F_{12}(t, u, v) d t\right) \\
& =\frac{1}{2 \pi}\left(-\frac{\alpha}{n}, \beta\right)=(0,0) .
\end{aligned}
$$

Since the function $f_{1}(u, v)=(0,0)$ we shall apply the averaging theory of second order. So we first compute

$$
\begin{aligned}
\int_{0}^{t} F_{11}(s, u, v) d s= & -\int_{0}^{t} \frac{p_{1}(s) \sin (n s)}{n} d s \\
& -\frac{3}{12 n^{4}}\left(\left(b_{1} u^{2} n^{2}-2 a_{1} u v n^{2}+3 b_{1} v^{2}\right) \cos (n t)\right. \\
& +\left(b_{1} u^{2} n^{2}+2 a_{1} u v n^{2}-b_{1} v^{2}\right) \cos (3 n t) \\
& -4\left(\left(-a_{1} u^{2} n^{3}+a_{1} v^{2} n+2 b_{1} u v n\right) \sin ^{3}(n t)\right. \\
& \left.\left.+b_{1} n^{2} u^{2}+2 b_{1} v^{2}-a_{1} n^{2} u v\right)\right) \\
\int_{0}^{t} F_{21}(s, u, v) d s= & \int_{0}^{t} p_{1}(s) \cos (n s) d s \\
& +\frac{1}{12 n^{3}}\left(-n\left(a_{1} n^{2} u^{2}-2 b_{1} v u-a_{1} v^{2}\right)\right. \\
& \cdot(3 \cos (n t)+\cos (3 n t)-4) \\
& -3\left(2 a_{1} u v n^{2}+b_{1}\left(3 n^{2} u^{2}+v^{2}\right)\right) \sin (n t) \\
& \left.+\left(b_{1}\left(v^{2}-n^{2} u^{2}\right)-2 a_{1} n^{2} u v\right) \sin (3 n t)\right) \\
D_{(u, v)}\left(F_{1}(t, u, v)\right)= & \left(\begin{array}{l}
\frac{\partial F_{11}(t, u, v)}{\partial u} \frac{\partial F_{11}(t, u, v)}{\partial v} \\
\frac{\partial F_{21}(t, u, v)}{\partial u} \frac{\partial F_{21}(t, u, v)}{\partial v}
\end{array}\right)
\end{aligned}
$$

Now we are ready to compute the function

$$
f_{2}(u, v)=\left(f_{21}(u, v), f_{22}(u, v)\right)
$$

defined in the Appendix, and we get the functions $f_{21}(u, v)$ and $f_{21}(u, v)$ which appear in the statement of the theorem. 
Now, from Theorem 3 , we obtain that for every $\varepsilon \neq 0$ sufficiently small and for every simple zero $\left(u^{*}, v^{*}\right)$ of the system (7), i.e. satisfying that

$$
\left.\operatorname{det}\left(\begin{array}{ll}
\frac{\partial f_{21}(u, v)}{\partial u} & \frac{\partial f_{21}(u, v)}{\partial v} \\
\frac{\partial f_{22}(u, v)}{\partial u} & \frac{\partial f_{22}(u, v)}{\partial v}
\end{array}\right)\right|_{(u, v)=\left(u^{*}, v^{*}\right)} \neq 0
$$

there exists a periodic solution $(u(t, \varepsilon), v(t, \varepsilon))$ of the differential system (6) such that

$$
(u(0, \varepsilon), v(0, \varepsilon)) \rightarrow\left(u^{*}, v^{*}\right) \quad \text { when } \varepsilon \rightarrow 0 .
$$

Going back through the change of variables (5) the periodic solution $(u(t, \varepsilon), v(t, \varepsilon))$ of the differential system (6) becomes the periodic solution

$$
\begin{aligned}
& z(t, \varepsilon)=\cos (n t) u(t, \varepsilon)+\frac{1}{n} \sin (n t) v(t, \varepsilon), \\
& w(t, \varepsilon)=-n \sin (n t) u(t, \varepsilon)+\cos (n t) v(t, \varepsilon),
\end{aligned}
$$

of the differential system (4) such that

$$
(z(0, \varepsilon), w(0, \varepsilon)) \rightarrow\left(u^{*}, v^{*}\right) \quad \text { when } \varepsilon \rightarrow 0 .
$$

Finally, since $x=\varepsilon z$ the periodic solution $(z(t, \varepsilon), w(t, \varepsilon))$ of the differential system (4) provides the periodic solution

$$
x(t, \varepsilon)=\varepsilon\left(\cos (n t) u(t, \varepsilon)+\frac{1}{n} \sin (n t) v(t, \varepsilon)\right),
$$

of the Lienard differential equation (2) such that

$$
x(0, \varepsilon) \approx \varepsilon u^{*}+\mathcal{O}\left(\varepsilon^{2}\right) .
$$

Moreover, since $x^{\prime}=\varepsilon w$ the theorem follows.

Proof of Corollary 2. We shall apply the results of Theorem 1 to the Lienard differential equation of the statement of Corollary 2. So we compute the functions $f_{21}(u, v)$ and $f_{22}(u, v)$ defined just before the statement of Theorem 1, and we obtain

$$
\begin{aligned}
& \frac{-\left(u^{3}-7 u+6\right) n^{4}+\left(u^{3}-2 v u^{2}-\left(v^{2}+2\right) u+8 v-4\right) n^{2}-2 v^{3}+u\left(v^{2}-1\right)+2}{4 n\left(3 n^{4}+2 n^{2}-1\right)}, \\
& \frac{\left(7 v+u\left(2 u^{2}-v u-8\right)\right) n^{4}+v\left(u^{2}+2 v u-v^{2}-2\right) n^{2}+v^{3}-v}{4 n\left(3 n^{4}+2 n^{2}-1\right)},
\end{aligned}
$$

respectively.

Doing the resultant of the functions $f_{21}$ and $f_{22}$ with respect to the variable $v$ we obtain a cubic polynomial in the variable $u$ which has the following three roots

$$
u_{1}=1, \quad u_{2}=2, \quad u_{3}=\frac{-3 n^{6}+31 n^{4}-25 n^{2}+5}{\left(n^{2}+1\right)^{3}} .
$$


In a similar way doing the resultant of the functions $f_{21}$ and $f_{22}$ with respect to the variable $u$ we obtain another cubic polynomial in the variable $v$ which has the following three roots

$$
v_{1}=1, \quad v_{2}=0, \quad v_{3}=\frac{-15 n^{6}+35 n^{4}-13 n^{2}+1}{\left(n^{2}+1\right)^{3}} .
$$

From the properties of the resultants it follows that all the solutions $\left(u^{*}, v^{*}\right)$ of the system $f_{21}(u, v)=0$, $f_{22}(u, v)=0$ are of the form $\left(u_{i}, v_{j}\right)$ being $u_{i}$ and $v_{j}$ some of the above roots. Trying the nine possible solutions, we obtain only two solutions for the system $f_{21}(u, v)=0, f_{22}(u, v)=0$, namely

$$
\left(u_{1}^{*}, v_{1}^{*}\right)=(1,1) \text { and }\left(u_{2}^{*}, v_{2}^{*}\right)=(2,0) .
$$

Then applying Theorem 1 the corollary follows.

For more information about the resultants see, for instance, Oliver (1999).

\section{ACKNOWLEDGMENTS}

The first author is partially supported by Ministerio de Economía y Competitividad (MiNECO) grant number MTM2013-40998-P, by Agència de Gestió d'Ajuts Universitaris i de Recerca (AGAUR) grant number 2013SGR-568, and by Marie Curie International Research Staff Exchange Scheme (IRSES) grants numbers FP7-PEOPLE-2012-IRSES 318999 and 316338. The second author is partially supported by Fundação de Amparo à Pesquisa do Estado de São Paulo (FAPESP) grant number 2015/02517-6. The third author is partially supported by FAPESP grant number 2012/18780-0. The three authors are also supported by Coordenação de Aperfeiçoamento de Pessoal de Nível Superior (CAPES) grant number 88881.030454/2013-01 from the program CSF-PVE. We thank to the referees for their helpful comments and suggestions.

\section{RESUMO}

Para $\varepsilon \neq 0$ suficientemente pequeno encontramos condições suficientes que garantem a existência de soluções periódicas para sistemas diferenciais de Lienard da forma

$$
x^{\prime \prime}+f(x) x^{\prime}+n^{2} x+g(x)=\varepsilon^{2} p_{1}(t)+\varepsilon^{3} p_{2}(t),
$$

onde $n$ é um inteiro positivo, $f: \mathbb{R} \rightarrow \mathbb{R}$ é uma função $C^{3}, g: \mathbb{R} \rightarrow \mathbb{R}$ é uma função $C^{4}$, e $p_{i}: \mathbb{R} \rightarrow \mathbb{R}$ para $i=1,2$ são funções contínuas $2 \pi$-periódicas. A principal ferramenta usada neste artigo é a teoria "averaging" de segunda ordem. Uma aplicação do resultado principal é feita.

Palavras-chave: solucão periódica, equação diferencial de Lienard, teoria da média, teoria de bifurcação.

\section{REFERENCES}

BUICĂ A AND LliBRE J. 2004. Averaging methods for finding periodic orbits via brouwer degree. Bull Sci Math 128: 7-22.

GINÉ J, GRAU M AND LLIBRE J. 2013. Averaging theory at any order for computing periodic orbits. Phys D 250: 58-65.

LLIBRE J. 2002. Averaging theory and limit cycles for quadratic systems. Radovi Matematicki 11: 215-228.

LLIBRE J, NOVAES DD AND TEIXEIRA MA. 2014. Higher order averaging theory for finding periodic solutions via brouwer degree. Nonlinearity 27: 563-583.

MA T AND WANG Z. 2013. Periodic solutions of lienard equations with resonant isochronous potentials. Disc Cont Dyn Sys 33: 1563-1581. 
OLIVER P. 1999. Classical invariant theory, volume 44 of London Math. Soc. Student Texts Cambridge Univ. Press, New York, $2^{\text {nd }}$ edition.

SANDERS JA, VERHULST F AND MURDOCK J. 2007. Averaging methods in nonlinear dynamical systems, volume 59 of Applied Mathematical Sciences Springer, New York, $2^{\text {nd }}$ edition.

\section{APPENDIX \\ THE AVERAGING THEORY OF SECOND ORDER}

In this section we recall the averaging theory of second order to find periodic orbits.

Theorem 3. Consider the differential system

$$
\dot{x}(t)=\varepsilon F_{1}(t, x)+\varepsilon^{2} F_{2}(t, x)+\varepsilon^{3} R(t, x, \varepsilon),
$$

where $F_{1}, F_{2}: \mathbb{R} \times D \rightarrow \mathbb{R}^{n}, R: \mathbb{R} \times D \times\left(-\varepsilon_{f}, \varepsilon_{f}\right) \rightarrow \mathbb{R}^{n}$ are continuous functions, T-periodic in the first variable, and $D$ is an open subset of $\mathbb{R}^{n}$. Assume that the following hypothesis (i) and (ii) hold.

(i) $F_{1}(t, \cdot) \in C^{1}(D)$ for all $t \in \mathbb{R}, F_{1}, F_{2}, R$ and $D_{x} F_{1}$ are locally Lipschitz with respect to $x$. We define $f_{1}, f_{2}: D \rightarrow \mathbb{R}^{n}$ as

$$
\begin{aligned}
& f_{1}(z)=\frac{1}{2 \pi} \int_{0}^{T} F_{1}(s, z) d s \\
& f_{2}(z)=\frac{1}{2 \pi} \int_{0}^{T}\left[D_{z} F_{1}(s, z) \int_{0}^{s} F_{1}(t, z) d t+F_{2}(s, z)\right] d s .
\end{aligned}
$$

(ii) For $V \subset D$ an open and bounded set and for each $\varepsilon \in\left(-\varepsilon_{f}, \varepsilon_{f}\right) \backslash\{0\}$, there exist $a \in V$ such that $f_{1}(a)+\varepsilon f_{2}(a)=0$ and $d_{B}\left(f_{1}+\varepsilon f_{2}, V, 0\right) \neq 0$ (see its definition later on).

Then for $|\varepsilon|>0$ sufficiently small, there exists a T-periodic solution $\varphi(\cdot, \varepsilon)$ of the system such that $\varphi(0, \varepsilon) \rightarrow a$ when $\varepsilon \rightarrow 0$.

As usual we have denoted by $d_{B}\left(f_{1}+\varepsilon f_{2}, V, 0\right)$, the Brouwer degree of the function $f_{1}+\varepsilon f_{2}: V \rightarrow \mathbb{R}^{n}$ at its fixed point $a$. A sufficient condition for showing that the Brouwer degree of a function $f$ at its fixed point $a$ is non-zero, is that the Jacobian of the function $f$ at $a$ (when it is defined) is non-zero.

If the function $f_{1}$ is not identically zero, then the zeros of $f_{1}+\varepsilon f_{2}$ are mainly the zeros of $f_{1}$ for $\varepsilon$ sufficiently small. In this case Theorem 3 provides the so-called averaging theory of first order.

If the function $f_{1}$ is identically zero and $f_{2}$ is not identically zero, then the zeros of $f_{1}+\varepsilon f_{2}$ are the zeros of $f_{2}$. In this case Theorem 3 provides the so-called averaging theory of second order. 